Conclusions In orthotopic mouse models of HCC, the antitumor activity of CMP-001 intravenous was greater than PD-1 blockade and comparable to sorafenib. CMP-001 intravenous was more active than CMP-001 subcutaneous in this model, which we hypothesize is due to increased liver exposure with intravenous infusion. Antitumor activity of CMP-001 monotherapy may be increased by combining it with standard of care or other therapies, as observed relative to historical benchmarks in ongoing CMP-001 clinical trials in patients with melanoma. CMP-001 intravenous may be a promising treatment option for patients with primary or metastatic liver cancers.

Acknowledgements This work was supported by Checkmate Pharmaceuticals. Studies were performed at Oncodesign Biotechnology (Dijon, France) and Crown Bioscience UK Ltd (Osgathorpe, UK) and National Research Council Canada (Ottawa, Ontario, Canada) and funded by Checkmate Pharmaceuticals.

Ethics Approval At Oncodesign Biotechnology, animal housing and experimental procedures were conducted according to French and European Regulations and the National Research Council Guide for the Care and Use of Laboratory Animals. The animal facility is authorized by the French authorities (Dijon: Agreement B21231011EA). The study and all animal procedures were approved by the Institutional Animal Care
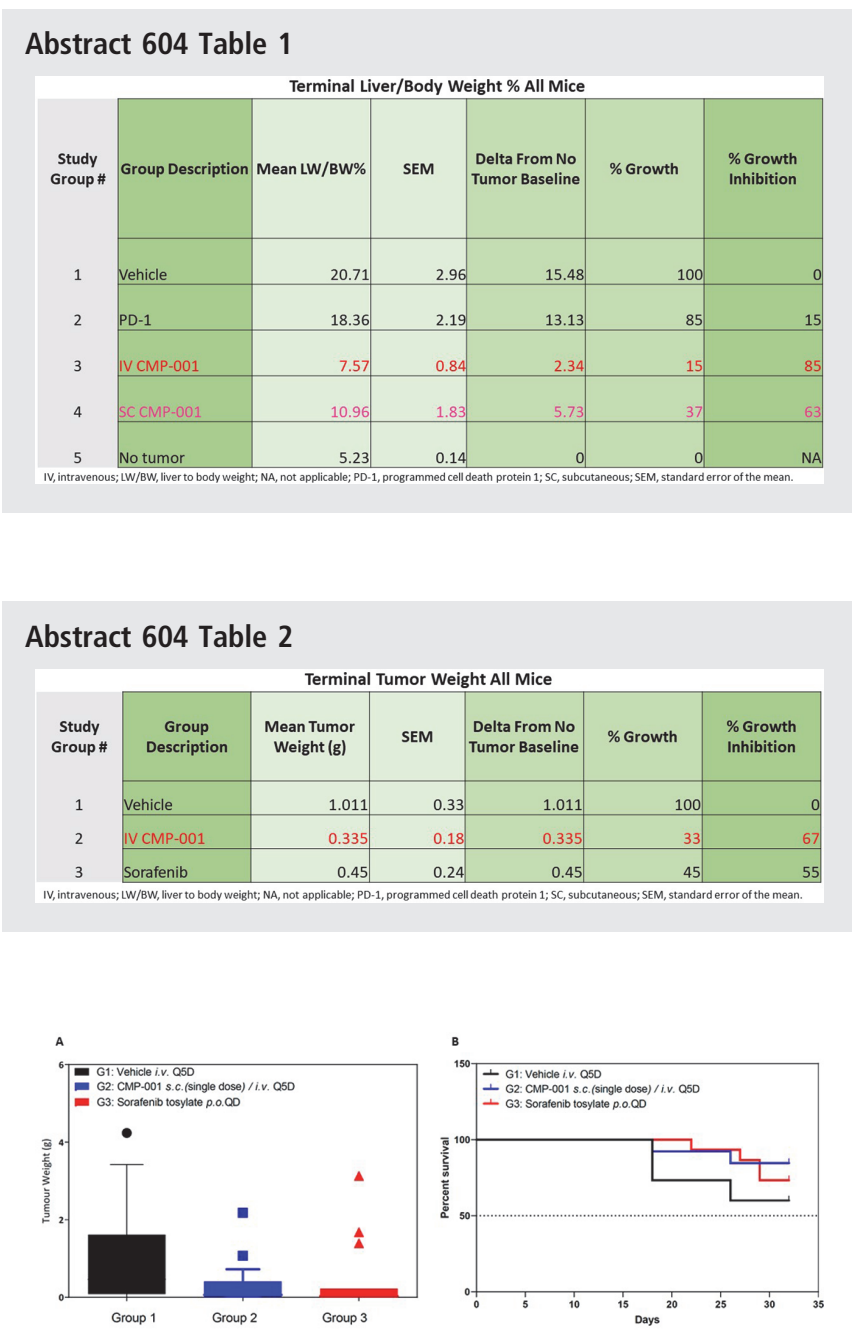

Abstract 604 Figure 1 and Use Committee of Oncodesign (Oncomet) approved by French authorities (CNREEA agreement number 91). At Crown Bioscience, animal care and experimental procedures were compliant with the UK Animals Scientific Procedures Act 1986 (ASPA) in line with Directive 2010/63/EU of the European Parliament and the Council of 22 September 2010 on the protection of animals used for scientific purposes. At National Research Council Canada, animals were maintained in accordance with the guidelines of the Canadian Council on Animal Care, and all experimental procedures were performed in accordance with regulations and guidelines reviewed and approved by the NRC Human Health Therapeutics Ottawa Animal Care Committee.

http://dx.doi.org/10.1136/jitc-2020-SITC2020.0604

\section{SYSTEMICALLY ADMINISTERED HER2-TARGETED ISACS PROVOKE A RAPID, LOCAL RESPONSE THAT ENGAGES THE INNATE AND ADAPTIVE ARMS OF THE IMMUNE SYSTEM TO ERADICATE TUMORS IN PRECLINICAL MODELS}

Heidi LeBlanc*, Cecelia Pearson, Justin Kenkel, Llsa Blum, Po Ho, Angela Luo, Richard Laura, Matthew Zhou, Joshua Gregorio, Andrew Luo, Shelley Ackerman, Brian Safina, David Dornan, Michael Alonso, Marcin Kowanetz. Bolt Biotherapeutics

Background Immune-stimulating antibody conjugates (ISACs) covalently attach immune stimulants to tumor-targeting antibodies such as trastuzumab. We have shown that HER2-targeted TLR7/8 ISACs elicit robust myeloid activation and tumor eradication in a TLR- and Fc-dependent manner in trastuzumab-resistant and HER2-low models. Upon treatment with ISACs, T cell-mediated immunological memory extends to tumor antigens beyond HER2. ${ }^{1}$ Here we describe the ISAC mechanism of action in vivo that leads to eradication of tumors in mice.

Methods Established syngeneic rHER2- or xenograft HER2expressing tumors treated with anti-HER2 ISACs or appropriate controls were assessed for gene expression by NanoString Pan-Cancer Immune Profiling panel comprising 750 genes related to tumor immune biology. Tumor cytokines were measured using MesoScale Discovery (MSD) technology, and immune cell infiltrates were assessed by immunohistochemistry (IHC). Anti-tumor efficacy was assessed after depletion of CD8 + T cells and phagocytes.

Results Within 24 hours of administration, HER2-directed ISACs induced robust, target-dependent activation of the immune system. In a syngeneic tumor model, 34\% of the measurable genes were significantly upregulated after treatment with the rHER2-targeted ISAC vs $0.1 \%$ with the non-binding ISAC control. Similarly, $13 \%$ vs $0 \%$ of genes were upregulated in a xenograft model after HER2-targeted vs control ISAC treatment. In both models anti-HER2 ISAC treatment led to activation of pathways indicative of TLR7/8 agonism (e.g. IRF-7; type 1 interferons), and FcgR engagement (e.g. NFkappaB associated genes). Cytokines and chemokines, including myeloid chemokines CCL2/3/4 and T cell chemokines CXCL9/ $10 / 11$ were specifically upregulated in the tumors at the gene and protein level, indicating robust activation of myeloid cells following anti-HER2 ISAC treatment. Furthermore, in syngeneic tumors $\mathrm{T}$ cell activation markers (e.g. Granzyme B; IFNgamma) were induced within 24 hours post treatment with an 
anti-rHER2 ISAC, and IHC at day 6 showed a 5 -fold increase in CD11c + cells. Control-treated tumors had sparse CD8 $+\mathrm{T}$ cells, whereas rHER2-targeted ISAC treatment led to $\sim 3.5$ fold increase in $\mathrm{T}$ cell frequency that shifted the tumor microenvironment from immunologically cold to hot. The recruitment of both phagocytes and $\mathrm{CD} 8+\mathrm{T}$ cells was consequential, as depletion of either abrogated anti-tumor efficacy of the rHER2-targeted ISAC. Systemically delivered ISACs were well-tolerated.

Conclusions In contrast to other immune therapies, such as anti-PDL1/PD1 and anti-CD40, systemically administered ISACs locally engage both the innate and adaptive arms of the immune system to eradicate tumors. The molecular and cellular phenotype associated with ISAC-mediated activation is being evaluated in the on-going BDC-1001 Phase I/II clinical trial. $^{2}$

\section{REFERENCE}

1. Ackerman S et al, Poster\# P756, SITC 20192. Phase 1/2 Study of BDC-1001 as a Single Agent and in Combination With Pembrolizumab in Patients With Advanced HER2-Expressing Solid Tumors; ClinicalTrials.gov (NCT04278144)

http://dx.doi.org/10.1136/jitc-2020-SITC2020.0605

\section{EPIDERMAL GROWTH FACTOR MODULATION OF CXCL1O IN KERATINOCYTES AND CUTANEOUS CANCERS}

${ }^{1}$ Myles McCrary*, ${ }^{1}$ David Gibbs, ${ }^{1}$ Carlos Moreno, ${ }^{2}$ Brian Pollack. ${ }^{1}$ Emory University School of Medicine, Decatur, GA, USA; ${ }^{2}$ Atlanta Veterans Affairs Medical Center, Decatur, GA, USA

Background Epidermal growth factor (EGF) signaling has wellestablished roles in cellular proliferation in normal tissue homeostasis and tumorigenesis. EGF receptor inhibitor therapy is associated with the development of a papulopustular rash and other cutaneous inflammatory effects. ${ }^{1}{ }^{2}$ These dosedependent toxicities are linked to treatment response and survival, and may reflect the interplay between EGF and the immune response. ${ }^{3} 4$ However, the effects of EGF signaling on inflammation in the skin and elsewhere are not entirely understood. ${ }^{6}$ In this study, we aimed to elucidate the immunomodulatory role of EGF in human keratinocytes exposed to the proinflammatory cytokine interferon- $\gamma$ (IFN- $\gamma$ ).

Methods Human keratinocyte cell lines (HaCaT) were exposed to IFN- $\gamma$, EGF, or both (48 hours). Differential gene expression analyses of RNA expression was performed using DESeq2. ${ }^{7}$ Fold change in gene expression on the $\log 2$ scale were calculated for each experimental treatment group relative to control. Web Gestalt was used to identify differentially expressed biologic pathways and gene networks, and further investigated in publicly available cutaneous squamous cell (cSCC) cell lines (GSE98767) and cSCC and basal cell carcinoma (BCC) tumor samples (GSE125285). ${ }^{8}$

Results As compared to untreated control cells, 2792 genes were differentially expressed following IFN- $\gamma$ treatment, 938 following EGF treatment, and 1248 following the combination of IFN- $\gamma$ and EGF (figure 1). To assess the impact of EGF on the cellular response to IFN-g, we identified IFN-g-induced genes whose expression was significantly altered by EGF (figure 2). We found that the induction of CXCL10 by IFN-g was among those significantly attenuated in the presence of EGF (padjusted $=0.01$ ) and selected CXCL10 as a model to further define the impact of EGF on immune gene expression. We found that in cutaneous SCC (cSCC) cell lines as well as cSCC and basal cell carcinoma tumor samples, the correlation between IFN- $\gamma$ and CXCL10 expression was abrogated in samples with higher EGF expression (figure 3).

Conclusions EGF has pleotropic roles in cancer including immunologic effects relevant to anti-tumor immunity. These studies demonstrate that EGF alters the transcriptional response to IFN-g including the induction of CXCL10 by IFN-g. Moreover, these studies suggest that in the setting of high EGF levels, there is a modulation of IFN-g-regulated chemokine expression. Further research is needed to clarify the
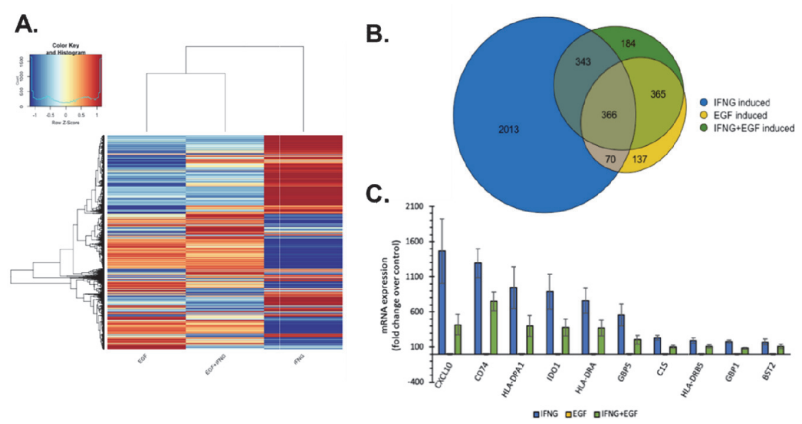

Abstract 606 Figure 1 EGF modulates IFN- $\gamma$-induced gene expression in human keratinocytes. A. Heatmap showing differentially expressed genes (Padjusted $<0.01$ ) induced by IFN- $\gamma$ alone, EGF alone, or IFN- $\gamma$ plus EGF (excluding genes that were not differentially expressed in any treatment group relative to control). B. Venn-diagram showing differentially expressed genes (Padjusted $<0.01$ ) induced by IFN- $\gamma$ and/ or EGF. C. Fold change of the top 10 genes induced after treated with IFN- $\gamma$ alone. The top 10 genes which were induced by IFN- $\gamma$ include CXCL10, CD74, several HLA-D genes, ID01, GBP5, C1S, and BST2

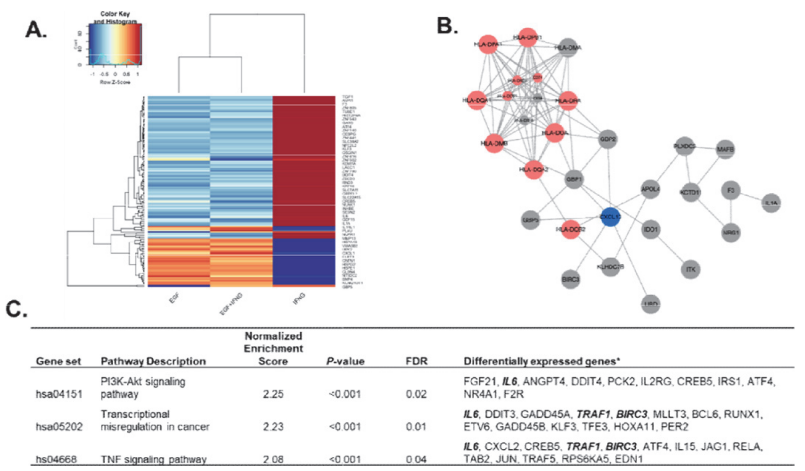

Abstract 606 Figure 2 Pathway analysis of genes induced by IFN- $\gamma$ then differentially regulated by EGF. A. Heatmap showing log2fold change in gene expression of top IFN- $\gamma$-regulated genes whose expression was significantly dampened or augmented by EGF (Pinteraction $<0.05$ ). The EGF* IFN- $\gamma$ interaction fold-change (far left) column indicates the excess fold change due to interaction between EGF and IFN- $\gamma$. Within this column, blue and red shading indicates dampening and augmentation of IFN- $\gamma$-induced gene expression by EGF, respectively. B. Sub-network graph from Network Topology Analysis (NTA) of IFN- $\gamma$-regulated genes of which expression was either 2 -fold higher or lower when EGF was added; genes in the top enriched GO Biological Process category are highlighted in red (G0:0019886 [antigen processing and presentation of exogenous peptide antigen via $\mathrm{MHC}$ class II]; Padjusted $=2.65 \times 10^{-7}$ ); blue shading of CXCL10 denoting it as the most strongly upregulated gene by IFN- $\gamma$ in this gene set to be dampened by EGF treatment. C. IFN- $\gamma$-induced genes attenuated by EGF, clustered according to significantly enriched KEGG pathways. Differentially expressed genes are listed in order of their score within the gene set enrichment analyses. Bolded italics type indicates common genes in multiple enriched pathways 